\title{
EFICIÊNCIA DAS FUNÇÕES WEIBULL E HIPERBÓLICA PARA DESCRIÇÃO DE DISTRIBUIÇÕES DIAMÉTRICAS DE POVOAMENTOS DE Tectona grandis ${ }^{1}$
}

\author{
Daniel Henrique Breda Binoti², Helio Garcia Leite ${ }^{3}$, Daniel Pereira Guimarães ${ }^{4}$, Mayra Luiza Marques da \\ Silva², Silvana Lages Ribeiro Garcia ${ }^{5}$ e Leonardo Pereira Fardin ${ }^{6}$
}

\begin{abstract}
RESUMO - As funções de densidade probabilidade Weibull e Hiperbólica foram comparadas quanto a eficiência de descrever a estrutura diamétrica de povoamentos de Teca (Tectona grandis L. f.) submetidas a desbaste. As duas funções com três e quatro parâmetros, foram ajustadas com dados de 98 parcelas permanentes, retangulares $\left(490 \mathrm{~m}^{2}\right)$, instaladas em um povoamento desbastado de Tectona grandis, no Estado do Mato Grosso e medidas durante 10 anos. Os ajustes foram feitos por Máxima Verossimilhança e a aderência foi avaliada pelo teste Kolmogorov-Smirnorv $(\alpha=1 \%)$. Também foram comparadas a soma de quadrados dos resíduos (SQR) dos diferentes ajustamentos. Todas as funções apresentaram aderência aos dados pelo teste de Kolmogorov-Smirnorv $(\alpha=1 \%)$. A função hiperbólica apresentou menor soma de quadrados de resíduos e menores valores para o teste de aderência. Foi possível concluir que a função hiperbólica foi mais eficiente para descrever a estrutura diametrica dos povoamentos estudados.
\end{abstract}

Palavras-chave: Distribuição diamétrica, Weibull, Hiperbólica e Teca.

\section{EFFICIENCY OF THE WEIBULL AND HYPERBOLIC FUNCTIONS FOR DESCRIBING THE DIAMETRIC DISTRIBUTIONS OF Tectona grandis STANDS}

\begin{abstract}
The Weibull probability density functions and Hyperbolic were compared by efficiency for describing the diameter structure of teak stands (Tectona grandis $L$. f.) undergoing thinning. These functions with three and four parameters were adjusted using data from 98 permanent plots and rectangular $\left(490 \mathrm{~m}^{2}\right)$, located in thinning stand of Tectona grandis in the Brazilian state of Mato Grosso, being measured for 10 years. Adjustments were made by Maximum Likelihood and adherence was assessed by Kolmogorov-Smirnorv test ( $\alpha=1 \%)$ and also compared to the residual squares sum (RSS) of the various adjustments. All of the functions presented adherence to data by the Kolmogorov-Smirnorv test $(\alpha=1 \%)$. The hyperbolic function had lower squares sum of waste and lower values for the adherence test. It was concluded that the hyperbolic function was more efficient to describe the diameter structure of the studied stands.
\end{abstract}

Keywords: Diameter distribution, Weibull, Hyperbolic and Teak.

\section{INTRODUÇÃO}

A madeira de teca (Tectona grandis L. f.) é considerada de alta qualidade, sendo destaque no mercado mundial, principalmente por causa das propriedades físico-mecânicas e das inúmeras possibilidades de uso. É utilizada em carpintaria, marcenaria, produção de móveis e construção naval (LAMPRECHT, 1990). As estimativas de preços para o mercado internacional para 2015 variam entre US $\$ 1.480,00$ e US $\$ 1.850$ por $\mathrm{m}^{3}$, dependendo da classe diamétrica (OLIVEIRA et al., 2007). Os povoamentos implantados com objetivos comerciais são manejados com a aplicação

\footnotetext{
${ }^{1}$ Recebido em 01.09.2008 e aceito para publicação em 16.12.2010.

${ }^{2}$ Programa de Pós-Graduação em Ciência Florestal pela Universidade Federal de Viçosa, UFV, Brasil. E-mail: $<$ danielbinoti@yahoo.com.br>.

${ }^{3}$ Universidade Federal de Viçosa, UFV, Brasil. E-mail: <hgleite@gmail.com>.

${ }^{4}$ Centro Nacional de Pesquisa de Milho e Sorgo, Embrapa. E-mail: <daniel@cnpms.embrapa.br>.

${ }^{5}$ Faculdade de Viçosa, FDV, Brasil. E-mail: < garciaslr@yahoo.com.br>.

${ }^{6}$ Floresteca, Brasil. E-mail: <leonardo.fadin@floresteca.com.br>.
} 
de desbaste, com ciclos médios entre 20 e 25 anos, visando obter, no corte final, distribuição diamétrica com árvores em classes superiores.

As funções de densidade probabilidade (f.d.p.) são utilizadas na área florestal para descrever a estrutura diamétrica do povoamento (MALTAMO et al., 2000). Isso permite quantificar multiprodutos, proceder a avaliações econômicas de conversão de árvores em multiprodutos e, também, simular desbastes (BURKHART et al., 1981).

Diversos tipos de distribuições são usados para descrever a estrutura diamétrica de um povoamento, entre os quais se destacam: distribuição gama (NELSON, 1964), log-normal (BLISS e REINKER, 1965), beta

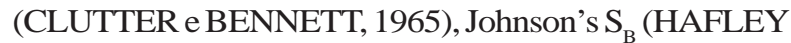
e SCHUREUDER, 1977) e distribuição Weibull, a qual foi descrita inicialmente por Weibull (1951) e utilizada no setor florestal por Bailey e Dell (1973). Desde então, a função Weibull tem sido amplamente difundida e utilizada na área florestal (CLUTTER eALLISON, 1974; HAFLEY e SCHREUDER, 1977; MATNEY e SULLIVAN, 1982). O método de ajuste dessas funções mais utilizado é o da máxima verossimilhança.

Segundo Guimarães (1994) e Eisfeld et al. (2005), a função Weibull apresenta superioridade na descrição da estrutura diamétrica de povoamentos florestais, quando comparada com as demais funções, contudo há estudos utilizando outras funções como a função Beta (SCHNEIDER et al., 1998) e a função Sb Johnson (SCOLFORO, 2003).

Leite et al. (2006) estudaram a função Weibull para a descrição da distribuição diamétrica de povoamentos de Tectona grandis submetidos a desbaste, sendo, posteriormente, esse trabalho avaliado por Nogueira et al. (2006) e confirmada a eficiência dessa função nesses tipos de povoamentos.

Em 2002, Guimarães propôs uma nova função (f.d.p.) para descrever distribuições de diâmetros definidas como função hiperbólica (equação 1). Tal função gera distribuições com pontos de inflexão, variando desde zero até o limite superior definido por tanh $(1)=0,76$. Isso confere a essa função maior flexibilidade quando comparado com a função Weibull, cujos pontos de inflexão variam entre zero e o limite de $(1-1 / e)=0,63$. A função hiperbólica proposta por Guimarães é definida por:

Revista Árvore, Viçosa-MG, v.35, n.2, p.299-306, 2011

$$
f(x)=\left\{\begin{array}{l}
\beta \gamma x^{(\gamma-1)}\left(1-\tanh \left(\beta x^{\gamma}\right)^{2}\right) \\
x, \beta, \gamma,>0
\end{array}\right.
$$

Neste estudo foi feita comparação entre as funções Weibull e Hiperbólica, de três e quatro parâmetros, para estimar a distribuição de diâmetros em povoamentos de Tectona grandis submetidos a desbaste.

\section{MATERIAL E MÉTODOS}

\subsection{Dados}

Os dados utilizados neste estudo foram provenientes de 98 parcelas permanentes retangulares de $490 \mathrm{~m}^{2}$ de área, instaladas em povoamentos de Tectona grandis, no Estado do Mato Grosso. Esses povoamentos foram implantados na região da depressão cuiabana, entre as coordenadas geográficas $15^{\circ} 02^{\prime}$ a $15^{\circ} 11^{\prime}$ de latitude $S$ e $56^{\circ} 29^{\prime}$ a $56^{\circ} 35^{\prime}$ de longitude $\mathrm{W}$, com espaçamento inicial de 3,0 x 2,0 $\mathrm{m}$. A precipitação média anual da região foi de 1.300 a $1.600 \mathrm{~mm}$, com seis meses de período seco, com a temperatura média anual de $25,3^{\circ} \mathrm{C}$. Um resumo dos dados é apresentado na Tabela 1.

As parcelas foram medidas aos 34, 43, 55, 68, 81, 92, 104, 120, 134 e 145 meses, em média. Em cada parcela e ocasião foram medidos os diâmetros à 1,30 m de altura (dap), de todas as árvores. Um primeiro desbaste foi realizado aos 81 meses (idade média) e um segundo aos 104 meses, com redução sistemática de 36\% das árvores em cada desbaste.

\subsection{Descrição das Funções}

As funções ajustadas para cada parcela e medição são descritas a seguir:

\subsection{Função Weibull}

A função Weibull de quatro parâmetros pode ser descrita como:

$f(X)=\left\{\begin{array}{l}\varpi\left(\frac{\gamma}{\beta}\right)\left(\frac{(x-\alpha)}{\beta}\right)^{(\gamma-1)} e^{-\left(\frac{(x-\alpha)}{\beta}\right)^{\gamma}}, \text { para } \alpha \leq x \leq \infty \\ 0, \text { para outros valores de } \mathrm{x}\end{array}\right.$

em que $\alpha$ é o parâmetro de locação, $\beta$ o parâmetro de escala $(\beta>0)$, $\gamma$ o parâmetro de forma $(\gamma>0)$, x o centro de classe de diâmetro $(x>0)$ e $\omega$ definido como o parâmetro assintótico, sendo o número de árvores da parcela. 
Tabela 1 - Características observadas em parcelas permanentes mensuradas em dez ocasiões em povoamentos de Tectona grandis localizados no estado do Mato Grosso, Brasil.

Table 1 - Observed characteristics in permanent plots measured on 10 occasions in Tectona grandis stands, State of Mato Grosso, Brazil.

\begin{tabular}{|c|c|c|c|c|c|c|c|}
\hline Idade & $\begin{array}{c}\text { Diâmetro } \\
\text { mínimo }(\mathrm{cm})\end{array}$ & $\begin{array}{c}\text { Diâmetro } \\
\text { máximo }(\mathrm{cm})\end{array}$ & $\begin{array}{l}\text { Altura total } \\
\text { média }(\mathrm{m})\end{array}$ & $\begin{array}{c}\text { Altura } \\
\text { dominante }(\mathrm{m})\end{array}$ & $\begin{array}{c}\text { Área Basal } \\
\left(\mathrm{m}^{2} \cdot \mathrm{ha}^{-1}\right)\end{array}$ & $\begin{array}{l}\text { Diâmetro médio } \\
\text { médio }(\mathrm{cm})\end{array}$ & $\begin{array}{c}\text { Número de } \\
\text { árvores por } \\
\text { hectare }\end{array}$ \\
\hline 34 & 6,4 & 11,7 & 8,7 & 9,5 & 9,63 & 9,4 & 1387 \\
\hline 43 & 7,3 & 14,7 & 9,4 & 11,5 & 14,16 & 11,4 & 1387 \\
\hline 55 & 10,3 & 17,2 & 12,1 & 13,5 & 19,85 & 13,5 & 1387 \\
\hline 68 & 12,9 & 19,7 & 14,3 & 15,0 & 27,54 & 15,9 & 1387 \\
\hline 81 & 15,2 & 21,5 & 15,9 & 17,5 & 13,12 & 18,1 & 510 \\
\hline 92 & 16,5 & 22,5 & 16,6 & 19 & 15,23 & 19,5 & 510 \\
\hline 104 & 18,3 & 25,8 & 17,9 & 19,5 & 11,74 & 22,1 & 306 \\
\hline 120 & 18,8 & 26,6 & 18,3 & 20,0 & 12,94 & 23,2 & 306 \\
\hline 134 & 21,7 & 30,2 & 20,2 & 21,7 & 16,75 & 26,4 & 306 \\
\hline 145 & 25,3 & 35,3 & 21,1 & 22,5 & 19,80 & 28,7 & 306 \\
\hline
\end{tabular}

A função Weibull de três parâmetros é resultante da supressão do parâmetro de locação e representada como:

$f(X)=\left\{\begin{array}{l}\varpi\left(\frac{\gamma}{\beta}\right)\left(\frac{(x)}{\beta}\right)^{(\gamma-1)} e^{-\left(\frac{(x)}{\beta}\right)^{\gamma}} \text {, para } x>0 \\ 0, \text { para outros valores de } \mathrm{x}\end{array}\right.$

A função Weibull com três e dois parâmetros é utilizada com frequência no setor florestal devido à sua flexibilidade, permitindo moldar diferentes tendências de distribuição, desde exponencial até normal. A correlação de seus parâmetros com atributos do povoamento e sua facilidade de ajustamento justificam o seu uso (GUIMARÃES, 1994; CAMPOS e LEITE, 2009).

\subsection{Função Hiperbólica}

A função Hiperbólica de quatro parâmetros descrita por Guimarães (2002) pode ser representada como:

$f(X)=\left\{\begin{array}{l}\varpi\left(\frac{\gamma}{\beta}\right)\left(\frac{(x-\alpha)}{\beta}\right)^{(\gamma-1)}\left(1-\tanh \left(\left(\frac{(x-\alpha)}{\beta}\right)^{\gamma}\right)^{2}\right), \text { para } \alpha \leq \mathrm{x} \leq \infty \\ 0, \text { para outros valores de } \mathrm{x}\end{array}\right.$

em que $\alpha$ é o parâmetro de locação, $\beta$ o parâmetro de escala $(\beta>0), \gamma$ o parâmetro de forma $(\gamma>0)$, x o centro de classe de diâmetro $(\mathrm{x}>0)$ e $\omega$ definido como o parâmetro assintótico, sendo o número de árvores da parcela.

A função Hiperbólica de três parâmetros pode ser expressa como:

$$
f(X)=\left\{\begin{array}{l}
\varpi\left(\frac{\gamma}{\beta}\right)\left(\frac{(x)}{\beta}\right)^{(\gamma-1)}\left(1-\tanh \left(\left(\frac{(x)}{\beta}\right)^{\gamma}\right)^{2}\right), \text { para } \mathrm{x}>0 \\
0, \text { para outros valores de } \mathrm{x}
\end{array}\right.
$$

\subsection{Ajuste das Funções}

Os dados de diâmetros observados em cada parcela e medição foram agrupados em classes com amplitude de $1,0 \mathrm{~cm}$. Utilizou-se em todas as funções um fator assintótico definido como o número de árvores da parcela, sendo o ajuste das funções feito pelo método de Máxima Verossimilhança (BALEY e DELL, 1973). Os ajustes foram feitos empregando o software kyplot, Versão 2.0, beta 15 (1997-2001c Koichi Yoshioka).

\subsection{Avaliação dos ajustes}

Para testar a aderência da função aos dados observados, foi aplicado o teste de Kolmogorov-Smirnorv (GIBBONS e SUBHABRATA, 1992; SOKAL e ROHLF, 1981) em um nível de $1 \%$ de probabilidade. Esse teste compara a frequência acumulada estimada com a frequência acumulada observada, sendo a classe de maior divergência a estatística do teste $(\mathrm{dn})$, definida por $d n=\operatorname{MAX}[F(X)-S(X)]$, em que: $\mathrm{S}(\mathrm{X})$ é a frequência acumulada observada e $F(X)$, a frequência estimada pela função densidade de probabilidade.

As estimativas de dn, juntamente com as somas de quadrados de desvios e com a análise gráfica de resíduos, foram utilizadas para comparar as funções ajustadas. Além disso, avaliou-se qual função apresentou a menor soma de quadrados dos desvios.

Revista Árvore, Viçosa-MG, v.35, n.2, p.299-306, 2011 


\section{RESULTADOS E DISCUSSÃO}

As médias das estimativas dos parâmetros das funções ajustadas são apresentadas na Tabela 1.

Cada ajuste foi comparado com a distribuição observada (Figura 1). Todos os ajustes resultaram em aderência aos dados pelo teste de KolmogorovSmirnorv $(\mathrm{P}>0,01)$. Na escolha da melhor função para descrição da estrutura diamétrica, somaram-se os valores de dn de cada ajuste para cada função, definindo a melhor função do menor valor de "dn total" e soma de quadrados dos resíduos totais (Tabela 2). A função hiperbólica apresentou menor soma de quadrado dos resíduos do que a função de Weibull (Tabela 2 e Figura 2). Considerando a soma dos quadrados dos desvios como parâmetro de comparação, a função Hiperbólica de quatro parâmetros apresentou melhores resultados do que a função Weibull em 65\% dos ajustes realizados.

Os gráficos de distribuição dos resíduos em função da idade do povoamento são apresentados na Figura 2. Na Figura 3 é apresentada a análise mais detalhada da distribuição de soma dos quadrados de resíduos.

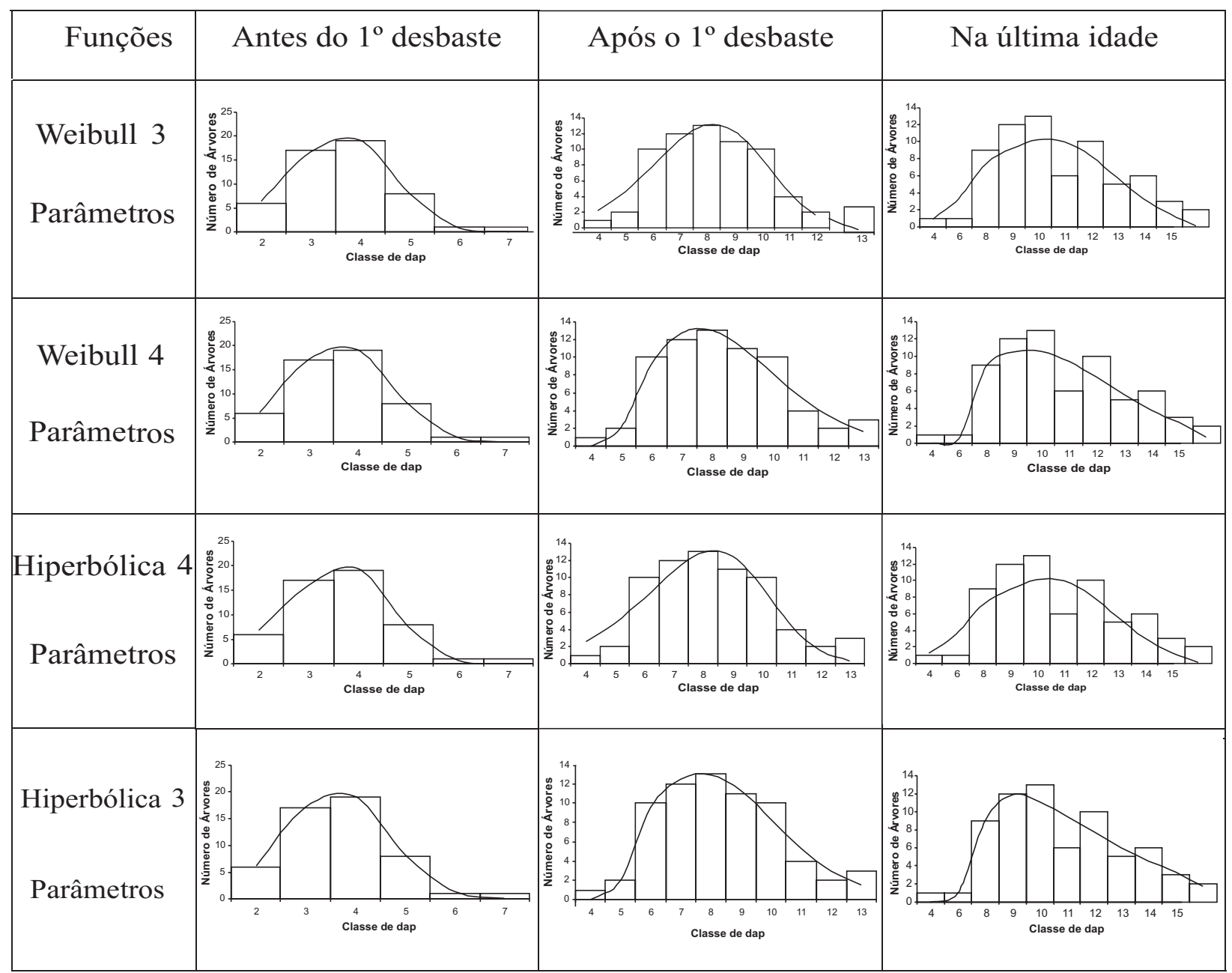

Figura 1 - Distribuições de diâmetro observadas (barras) e estimadas (linhas) das funções Weibull e Hiperbólica, com três e quatro parâmetros, para parcelas ao acaso (última medição antes do primeiro desbaste, primeira medição após desbaste e última medição).

Figure 1 - Observed (bars) and estimated (lines) diameter distributions of the Weibull and Hyperbolic Functions, with three and four parameters for randomized plots (last measurement before the first thinning, the first measurement after thinning, and final measurement).

Revista Árvore, Viçosa-MG, v.35, n.2, p.299-306, 2011 
A supressão do parâmetro de locação resultou em diminuição da exatidão no ajuste (Tabela 2). Isso indica que a desconsideração desse parâmetro deve ser feita somente quando acarretar diminuição na precisão do sistema de equações utilizado na sua projeção da distribuição diamétrica em idades futuras (GUIMARÃES, 1994).

Tabela 2 - Soma de Quadrado de Resíduos (SQR) e valores totais dos testes Kolmogorov-Smirnorv ( $d n$ ), obtidos a partir do ajuste das funções Weibull e Hiperbólica de 98 parcelas permanentes instaladas em plantios desbastados de Tectona grandis em idades entre 34 e 145 meses.

Table 2-Residual squares sum (RSS) and Kolmogorov-Smirnorv (dn) test total values for the studied functions obtained from the Weibull adjustment and Hyperbolic functions of 98 permanent plots set up in thinned Tectona grandis plantings, aged between 34 and 145 months.

\begin{tabular}{ccc}
\hline Função & SQR Total & dn Total \\
\hline Weibull 3 Parâmetros & 20550,72707 & 135,4980592 \\
Weibull 4 Parâmetros & 16323,70723 & 114,9780218 \\
Hyperbólica 3 Parâmetros & 20337,99505 & 126,5226101 \\
Hyperbólica 4 Parâmetros & 15906,16138 & 112,5778944 \\
\hline
\end{tabular}

\section{Função Hiperbólica com 4 parâmetros}

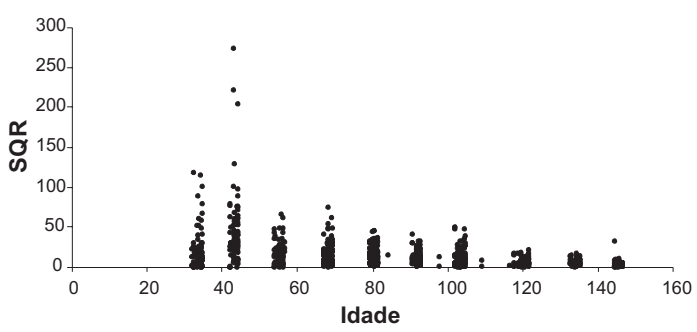

Função Weibull com 4 parâmetros

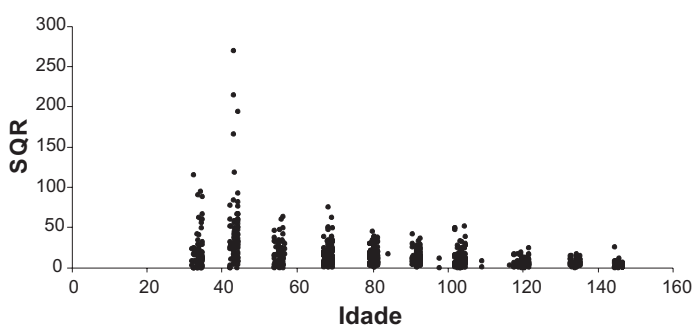

A função Hiperbólica de quatro parâmetros demonstrou-se melhor tanto pela soma de quadrados de resíduos quanto por apresentar menores valores de dn no teste de Kolmogorov-Smirnorv. A minimização do valor dn pode ser utilizada para a comparação das funções e a escolha da melhor descrição dos dados observados, como proposto por Scolforo e Thierschi (1998).

A função Weibull desde sua introdução no setor florestal tem sido empregada por diversos autores para modelagem de distribuições de diâmetros (BAILEY e DELL, 1973; MAGNUSSEN, 1986; GOVE e PATIL, 1998; KANGAS e MALTAMO, 2000; CAO, 2004; NOGUEIRA et al., 2006; CAMPOS e LEITE, 2006; METATALO et al., 2006).

A função hiperbólica apresenta grande potencial para a descrição da distribuição diamétrica de povoamentos de Tectona grandis desbastados devido à sua flexibilidade. $\mathrm{O}$ ponto de inflexão dessa função varia desde zero até o limite superior definido por $I=\tanh (1)=0.76$. Isso confere maior flexibilidade em relação à função Weibull, cujos pontos de inflexão

\section{Função hiperbólica com 3 parâmetros}

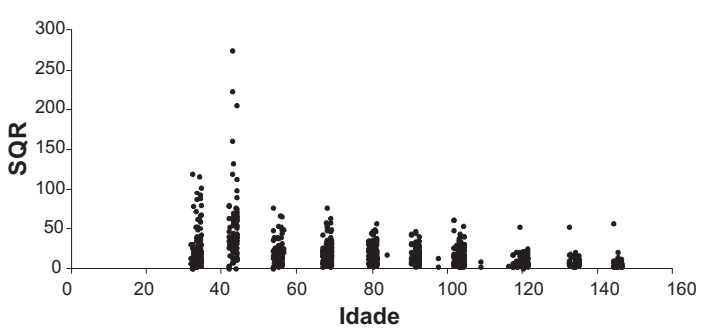

Função Weibull com 3 parâmetros

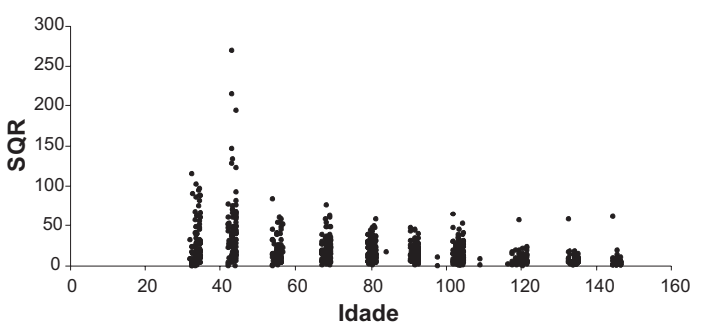

Figura 2 - Distribuição das soma de quadrados dos resíduos em relação à idade de medição das parcelas permanentes em povoamentos de Tectona grandis desbastados.

Figure 2 - Distribution of the Residual Sum of Squares in relation to the measuring age of permanent plots in thinned Tectona grandis stands 


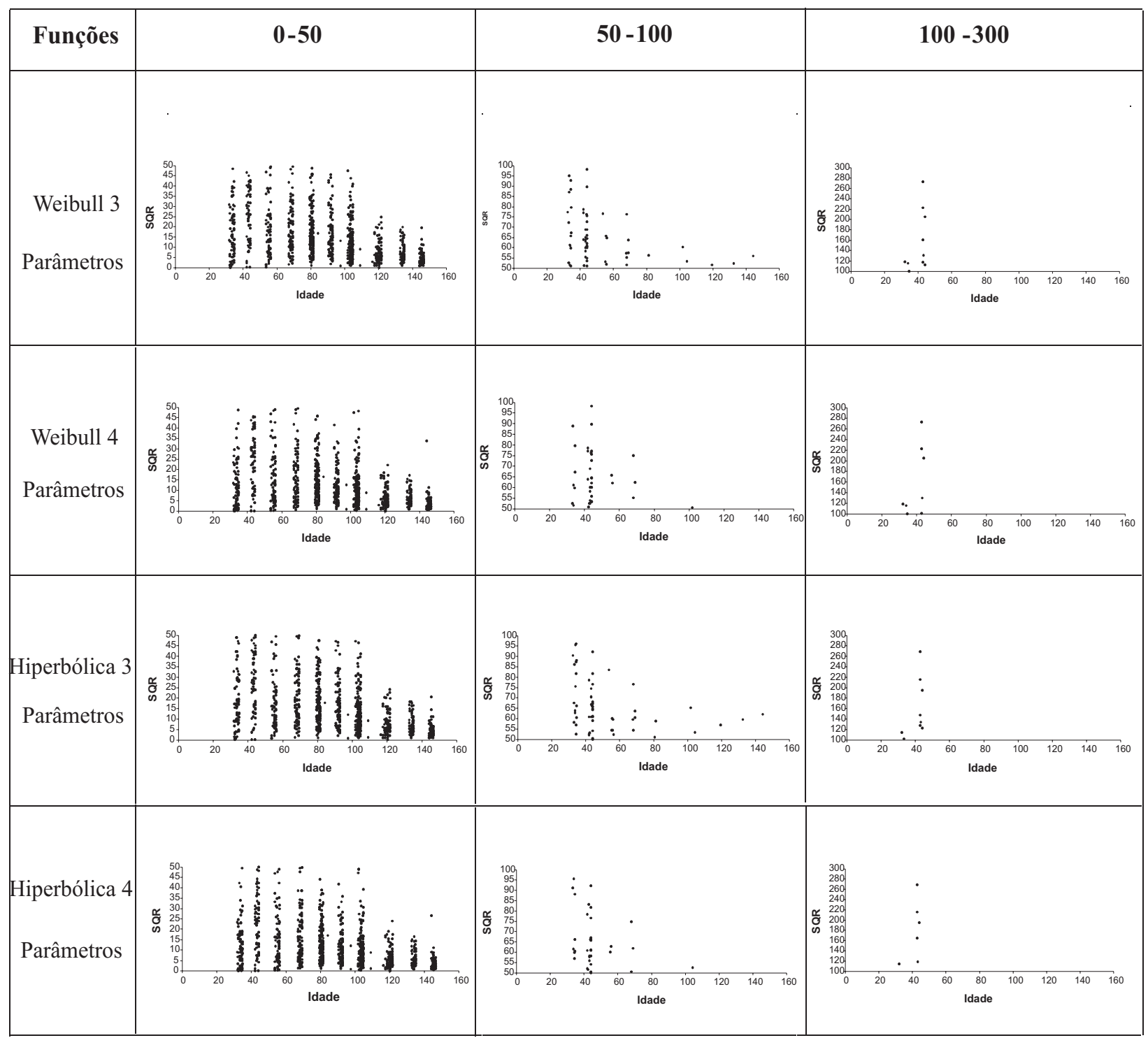

Figura 3 - Distribuição em faixas da soma de quadrados de resíduos em relação a idade de medição para 98 parcelas permanentes em povoamentos de Tectona grandis desbastados.

Figure 3 - Distribution in ranges of the Residual Sum Squares in relation to the measuring age of 98 permanent plots in thinned Tectona grandis stands.

variam entre zero e o limite de $I=(1-1 / e)=0.63$, possibilitando a função hiperbólica maior capacidade de descrição de diferentes estruturas diamétricas.

\section{CONCLUSÃO}

A função Hiperbólica é mais eficiente do que a de Weibull para estimar a distribuição diamétrica dos povoamentos estudados de Tectona grandis submetidos a desbaste.

\section{REFERÊNCIAS}

BAILEY, R. L.; DELL, T. R.. Quantifying diameter distributions with the Weibull function. Forest Science, v.19, n.2, p.97-104, 1973.

BLISS, C. L.; REINKER, K. A. A lognormal approach to diameter distributions in even-aged stands. Forest Science, v.10, p.350-360, 1965.

Revista Árvore, Viçosa-MG, v.35, n.2, p.299-306, 2011 
BURKHART, H. E.; CAO. Q. V.; WARE, Z. A. A comparison of growth and yield prediction models for loblolly pine. Blacksburg: Virginia Polytechnic Institute and State University, Scholl of Forestr and Wildlife Resources, 1981. 59p. (Publ., FWS-2).

CAMPOS, J. C. C.; LEITE, H. G. Mensuração florestal: perguntas e respostas. 3.ed. rev. e ampl.. Viçosa, MG: Universidade Federal de Viçosa, 2009. 548p.

CAO, Q. V. Predicting parameters of a Weibull function for modeling diameter distribution. Forest Science, v. 50, n. 4, p.682-685, 2004.

Clutter, J. L.; Allison, B. J. A growth and yield model for Pinus radiata in New Zealand for tree and stand simulation. Stockholm: Royal College of Forestry, 1974. p. 136160. 1974 (Research Notes, 30).

CLUTTER, J. L.; BENNETT, F.A. Diameter distributions in old - field slash pine plantations. Georgia Forest Research Council Report, n.13, p.1-9, 1965.

EISFELD, R. L. et al. Modelagem do crescimento e da produção de Pinus taeda L. por meio de função probabilística. Floresta, v.35, n.2, p.317-328, 2005.

GIBBONS, J. D.; SUBHABRATA, C.

Nonparametric statistical inference.

3.ed. New York: Marcel Dekker, 1992. 544p.

(Statistics: textbook and monograph, v.31)

GOVE, J. H.; PATIL, G. P. Modelling basal área size distribution of forest stands: a compatible approach. Forest Science, v.44, n.2, p.285-297, 1998.

GUIMARÃES, D. P. Desenvolvimento de um modelo de distribuição diamétrica de passo invariante para prognose e projeção da estrutura de povoamentos de eucalipto. 1994. 178f. Tese (Doutorado em Ciência Florestal) - Universidade Federal de Viçosa, Viçosa, MG, 1994.

GUIMARÃES, D. P. Uma função

hiperbólica de distribuição probabilística de alta flexibilidade. Planaltina: Embrapa Cerrados, 2002. 40p.
HAFLEY, W. L.; SCHREUDER, H. T. Statistical distributions for fitting diameter and height data in ever-aged stands. Canadian Journal of Forest Research, v.7, p.184-487, 1977.

KANGAS, A.; MALTAMO, M. Calibrating predicted diameter distribution with additional information. Forest Science, v.46, n.3, p.390-396, 2000.

LAMPRECHT, H. Silvicultura nos trópicos: ecossistemas florestais e respectivas espécies arbóreas possibilidades e métodos de aproveitamento sustentado. Eschborn: Deustsche Gessellschaft Für Technische Zusammenarbeit (GTZ) GmbH, 1990. 343p.

LEITE, H. G. et al. Um modelo de distribuição diamétrica para povoamentos de Tectona grandis submetidos a desbaste . Revista Árvore, v.30, n.1, p.89-98, 2006.

MAGNUSSEN, S. Diameter distribution in picea abies described by the weibull model.

Scandinavian Journal Forest Research, n.1, p.493-502, 1986

MALTAMO, M. et al. Comparison of percentile based prediction methods and the Weibull distribution in describing the diameter distribution of heterogeneous Scots pine stands. Forest Ecology and Management, v.133, p.263-274, 2000

MATNEY, T. G.; SULLIVAN, A. D.. Variable top volume and height predictions for slash pine trees. Forest Science, v.28, n.2, p.274-282, 1982.

MEHTÄTALO, L., MALTAMO, M.; KANGAS, A. The use of quantile trees in the prediction of the diameter distribution of a stand. Silva Fennica, v.40, n.3, p.501-516, 2006.

Revista Árvore, Viçosa-MG, v.35, n.2, p.299-306, 2011 
NELSON, T. C. Diameter distribution and growth of loblolly pine. Forest Science, v.10, n.1, p.105-114, 1964.

NOGUEIRA, G. S. et al. Avaliação de um modelo de distribuição diamétrica ajustado para povoamentos de Tectona grandis submetidos a desbaste. Revista Árvore, v.30, n.3, p.377-287, 2006.

OLIVEIRA, L. C.; ANGELI, A.; STAPE, J. L. Teca é a nova opção na indústria mundial. Revista da Madeira, v.18, n.106, p.81-86, 2007.

SCHNEIDER, P. R. et al. Produção de Eucalyptus grandis Hill ex Maiden em diferentes intensidades de desbaste. Ciência Florestal, n.8, p.129-140, 1998.
SCOLFORO, J. R. S. et al. Sb distribution accuracy to represent the diametric structure of Pinus taeda, through five methods. Forest Ecology and Management, v.175, n.1, p.489-496, 2003.

SCOLFORO, J. R. S.; THIERSCH, A. Estimativas e testes da distribuição de frequência diamétrica para Eucalyptus camaldulensis através da distribuição Sb, por diferentes métodos de ajuste. Scientia Florestalis, v.54, p.91-103, 1998.

SOKAL, R. R.; ROHLF, F. J. Biometry. San Francisco: Freeman, 1981. 859p.

WEIBULL, W.. A statistical distribution function of wide applicability. Journal of Applied Mechanics, v.18, p.293-297, 1951. 